\title{
Effects of testosterone replacement therapy on cortical and trabecular bone mineral density, vertebral body area and paraspinal muscle area in hypogonadal men
}

\author{
Eckhard Leifke, Hans-Christian Körner, Thomas M Link ${ }^{1}$, Hermann M Behre, Peter E Peters ${ }^{1}$ \\ and Eberhard Nieschlag \\ Institute of Reproductive Medicine, and ${ }^{1}$ Institute of Clinical Radiology of the University, D-48129 Münster, Germany \\ (Correspondence should be addressed to E Nieschlag, FRCP, Institute of Reproductive Medicine, Domagkstr. 11, D-48149 Münster, Germany)
}

\begin{abstract}
Loss of bone and muscle mass are major findings of male hypogonadism. In order to determine the long-term effect of testosterone replacement therapy on spinal bone and muscles, the trabecular and cortical bone mineral density, vertebral body area and paraspinal muscle area were assessed by quantitative computed tomography in 32 testosterone-substituted patients, aged 18-74 years, with idiopathic hypogonadotropic hypogonadism $(n=6)$, pituitary insufficiency $(n=5)$, Klinefelter syndrome $(n=12)$ or other forms of primary hypogonadism $(n=9)$. They were followed for a mean period of $3.2 \pm 1.7$ years (mean \pm S.D.), ranging from 1 to 7 years. A significant correlation between initial serum testosterone levels and bone mineral density was found in patients with congenital forms $(r=0.58 ; P<0.05)$ but not in those with acquired forms. A significant increase in trabecular and cortical bone mineral density $(P<0.001)$ was documented in the course of replacement therapy in all patients regardless of the type of hypogonadism and age of patients. A slight but significant increase in paraspinal muscle area was observed if all patients were taken together $(P<0.01)$. The area of paraspinal muscle correlated with body weight $(r=0.58 ; P<0.001)$ and moderately with trabecular bone mineral density $(r=0.4 ; P<0.01)$. Its increase did not correspond to the change observed for trabecular and cortical bone mineral density. Vertebral body area did not change over time. It correlated only with height and weight but not with bone mineral density. In conclusion, testosterone therapy of hypogonadal men improves both trabecular and cortical bone mineral density of the spine independently of age and type of hypogonadism while vertebral area remains unchanged. The effects seen on paraspinal muscles emphasize the clinical benefit of adequate replacement therapy for the physical fitness of hypogonadal men.
\end{abstract}

European Journal of Endocrinology 138 51-58

\section{Introduction}

In men, androgen deficiency leads to loss of bone and muscle mass. Physical fragility and bone fractures are the consequences $(1,2)$. Beneficial effects of testosterone replacement therapy on bones and muscles in hypogonadal men have been reported (3-8). Little is known, however, about whether the effects described for bone vary according to age and type of hypogonadism $(9,10)$ or potential changes in muscle mass. Furthermore, the responsiveness of cortical and trabecular bone to androgens may be different and may be obscured by differences in biomechanical covariates between appendicular and axial bone when assessed on different skeletal sites (see, for example (3)). In addition, most studies concerning bone in hypogonadal men have focused on bone mineral density (BMD) alone and did not take bone dimension into consideration (10). Both are, however, determinants of compressive strength $(11,12)$ and fracture risk $(13)$.

A relationship between muscle strength and size and BMD in athletes has been reported(14-16). Muscle mass also increases in hypogonadal men with testosterone therapy $(6,8)$. A recent study has shown that an increase in muscle mass was accompanied by a change in biochemical serum markers of bone formation and bone resorption (7). Thus a close relationship between diverse anabolic effects in hypogonadal men undergoing replacement therapy should be expected. Again, there is little information for spinal bone and muscles in hypogonadal men. Therefore we performed quantitative computed tomography (QCT) of the lumbar spine allowing simultaneous quantification of trabecular BMD (tBMD) and cortical BMD (cBMD), the cross-sectional area of the vertebral bodies and of the adjacent paraspinal muscles in 
hypogonadal men undergoing testosterone replacement therapy.

\section{Subjects and methods}

\section{Subjects and treatment}

Thirty-two hypogonadal men attending the Institute of Reproductive Medicine undergoing effective testosterone replacement therapy (17) and serial measurements of the lumbar spine BMD by QCT were included in the evaluation. Patients with morning serum testosterone below $12 \mathrm{nmol} / \mathrm{l}$ and normal to elevated luteinizing hormone (LH) and follicle-stimulating hormone (FSH) levels on two different occasions were classified as having primary hypogonadism, and patients with lowered serum testosterone $(<12 \mathrm{nmol} / \mathrm{l})$ and lowered basal $\mathrm{LH}(<1.5 \mathrm{U} / \mathrm{l})$ and FSH ( $<2 \mathrm{U} / \mathrm{l})$ and without stimulatory response to i.v. injection of gonadotrophin-releasing hormone $(\mathrm{GnRH})$ were diagnosed as suffering from secondary hypogonadism. The first QCT was performed before initiation of testosterone therapy. The patients suffered from Klinefelter syndrome (XXY; $n=12$ ), acquired forms of primary hypogonadism due to orchitis, trauma, orchiectomy or unknown causes (APH; $n=9$ ), idiopathic hypogonadotropic hypogonadism (IHH; $n=6)$ and acquired forms of secondary hypogonadism due to pituitary tumour (two craniopharygeoma and three prolactinoma) or hypophysectomy (ASH; $n=5)$. They were treated and followed up for $3.2 \pm 1.7$ years (means \pm S.D. ranging from 1 to 7 years). Patients' mean \pm S.D. age at the beginning of the study was $34 \pm 1.7$ years, ranging from 18 to 74 years. Thirtytwo patients were treated by i.m. injection of testosterone enanthate $(250 \mathrm{mg}$ Testoviron Depot; Schering, Berlin, Germany) (17). Seven patients with secondary hypogonadism were switched to human choriogonadotrophin/human menopausal gonadotrophin or $\mathrm{GnRH}$ for $1.1 \pm 0.6$ years to induce spermatogenesis and paternity (18). Other endocrine functions were regularly assessed and effectively replaced if required in patients with secondary hypogonadism. Patients had no other diseases and did not use medications that would interfere with bone metabolism.

\section{QCT}

All patients underwent scanning of the three vertebrae L-2 to L-4 using a standard QCT protocol. A tomoscan LX scanner (Phillips, Eindhoven, The Netherlands) was used. A lateral scanogram was performed and midvertebral sections were determined parallel to the end plates of the vertebrae. Regions of interest (ROIs) were determined by automated evaluation: a Pacman ROI was used in all images to ascertain that the ROI was the same for all patients and measurements. This procedure allows automated tracing of the vertebrae and permits separation of cortical and trabecular
BMD (19). A $\mathrm{K}_{2} \mathrm{HPO}_{4}$-calibration phantom placed under the patient's back was scanned simultaneously in all patients in order to extrapolate individual values to the same linear standard for all patients (20). BMD values for each subject were obtained by averaging the BMD values of the different vertebral levels and were expressed as $\mathrm{mg} \mathrm{K}_{2} \mathrm{HPO}_{4} / \mathrm{cm}^{3}$ density equivalents. A single energy technique (SE-QCT), the method of choice in longitudinal studies, was applied. Using automated ROI the coefficient of intrasubject variation is less than $4 \%$ (21). Reference values for tBMD and cBMD were used from a study by Kalender et al. (19), who measured $\mathrm{BMD}$ of the spine in 135 males (age ranging from 18 to 80 years) by SE-QCT. All were considered normal with respect to bone metabolism.

The area of the vertebrae in the midvertebral plane (MVA) as well as the area of the paraspinal muscles (PMA) were determined on an off-line image processing workstation with automated quantitative image evaluation software (Gyroview, Phillips). A standardized thresholding algorithm was used to define the anterior and lateral borders of the vertebrae. The posterior border was determined by a line parallel to the transverse processes; this was at a tangent to the central most ventral border of the posterior border of the vertebral body. As in the studies by Gilsanz et al. $(22,23)$, all structures behind the most anterior margin of the spinal canal, including the pedicle and the posterior elements, were excluded. Thus optimized reproducibility with an intrasubject coefficient of variation of less than $1 \%$ was obtained.

In order to determine the PMA, a standardized thresholding was used to segment the muscle from the surrounding fatty tissue. The precision of this procedure has been shown to vary by $2 \%(24)$. In order to optimize intrasubject variability for serial measurements, a line was drawn along the spinal and the left transverse process. Thus the area of the left paraspinal musculature was segmented and measured in all patients.

\section{Hormonal measurements}

Blood samples were drawn from all patients for testosterone measurements between 0800 and $1200 \mathrm{~h}$. Serum testosterone levels were measured by RIA as described previously (25). The detection limit for testosterone was $0.7 \mathrm{nmol} / \mathrm{l}$; the intra- and inter-assay variation were 6 and $8 \%$ respectively.

$\mathrm{FSH}, \mathrm{LH}$ and prolactin (PRL) in serum were measured in duplicate by immunofluorimetric assay using commercial kits (Delfia hFSH, Delfia hLH, Delfia PRL; ADL, Freiburg, Germany).

\section{Statistical analysis}

All parameters were tested for normality by the Kolmogorov-Smirnov test and, if not normally distributed, log-transformed and back-transformed for plotting. Comparisons between baseline and follow-up 
Table 1 Anthropometric parameters, serum testosterone levels before treatment, tBMD, and cBMD, MVA and PMA according to the type of hypogonadism.

\begin{tabular}{lccrr}
\hline & $\begin{array}{c}\mathbf{X X Y} \\
(n=12)\end{array}$ & $\begin{array}{c}\text { APH } \\
(n=9)\end{array}$ & $\begin{array}{c}\text { IHH } \\
(n=6)\end{array}$ & $\begin{array}{c}\text { ASH } \\
(n=5)\end{array}$ \\
\hline Age (years) & $35.3 \pm 5.2$ & $34.4 \pm 2.7$ & $33.3 \pm 6.5$ & $33.1 \pm 1.8$ \\
Height (cm) & $179 \pm 1.6$ & $177 \pm 2.8$ & $175 \pm 6.0$ & $184 \pm 2.1$ \\
Weight (kg) & $86.2 \pm 5.1$ & $85.7 \pm 6.2$ & $68.3 \pm 5.3$ & $82.7 \pm 6.9$ \\
Testosterone (nmol/l) & $5.6 \pm 1.6$ & $7.3 \pm 1.7$ & $3.1 \pm 1.8$ & $3.2 \pm 1.3$ \\
tBMD (mg/cm $\left.{ }^{3}\right)$ & $125 \pm 12$ & $117 \pm 13$ & $82 \pm 10$ & $112 \pm 20$ \\
cBMD (mg/cm $\left.{ }^{3}\right)$ & $276 \pm 31$ & $268 \pm 7$ & $221 \pm 27$ & $255 \pm 23$ \\
MVA $\left(\mathrm{cm}^{2}\right)$ & $14.1 \pm 0.5$ & $13.9 \pm 0.6$ & $12.4 \pm 1.5$ & $15.6 \pm 0.9$ \\
PMA $\left(\mathrm{cm}^{2}\right)$ & $23.7 \pm 1.3$ & $26.8 \pm 1.8$ & $20.8 \pm 1.9$ & $22.2 \pm 1.3$
\end{tabular}

$\mathrm{XXY}$, men with Klinefelter syndrome; $\mathrm{APH}$, men with acquired forms of primary hypogonadism; IHH, men with idiopathic hypogonadotropic hypogonadism; $\mathrm{ASH}$, men with acquired forms of secondary hypogonadism.

parameters were made by Student's paired $t$-test. Twotailed $P<0.05$ was taken as the level of significance. Raw data were plotted and linear regression was performed between initial and final values of vertebral area in order to obtain an estimate for the individual and overall change. In order to define a possible relationship between different parameters, bivariate correlation was performed. In addition, partial correlation was performed to control for possible covariates such as weight, height and age. Percentage changes were arcsinustransformed before correlations were performed. All tests were computed using a statistical software package (SSPS, Chicago, IL, USA). If not otherwise stated, data are given as means \pm S.E.M.

\section{Results}

\section{Initial values}

Data for initial serum testosterone, age, body height, weight, initial values for tBMD and cBMD, MVA and
PMA are listed in Table 1 according to the type of hypogonadism. Significant differences between the means could not be demonstrated because of the limited number of patients and low statistical power for ANOVA. Patients with IHH showed the lowest serum testosterone levels, tBMD, cBMD, MVA and PMA. Patients with Klinefelter syndrome (XXY) and APH showed higher tBMD and CBMD of the spine and had higher serum testosterone values. If all patients were taken together, tBMD was significantly correlated with cBMD $(0.69 ; P<0.001)$. A significant correlation between BMD and initial serum testosterone was only seen if patients with congenital forms of hypogonadism (XXY and IHH; $n=18$ ) were analysed separately $(r=0.58 ; P<0.05)$. There was no correlation when all patients were taken together. No correlation was found between the MVA and tBMD or cBMD even when partial correlation was controlled for weight, height and age. Midplane area of the vertebral bodies showed a significant correlation with body height $(0.58 ; P<0.01)$ and body weight $(r=0.41 ; P<0.05)$. Paraspinal area at
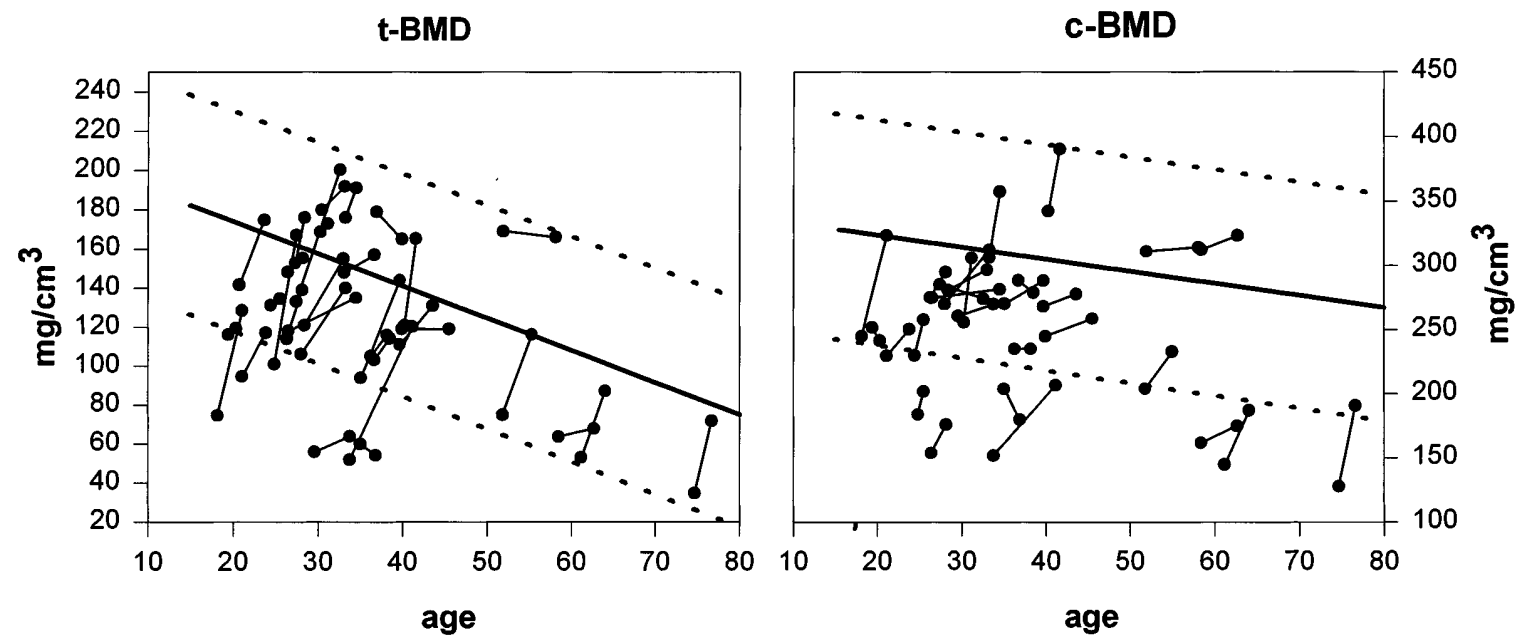

Figure 1 Age of all 32 hypogonadal men plotted against their initial tBMD or cBMD and $3.2 \pm 1.7$ years after initiation of testosterone treatment. The solid lines refer to the mean values for age, and dashed lines are \pm 2 S.D. (18). 


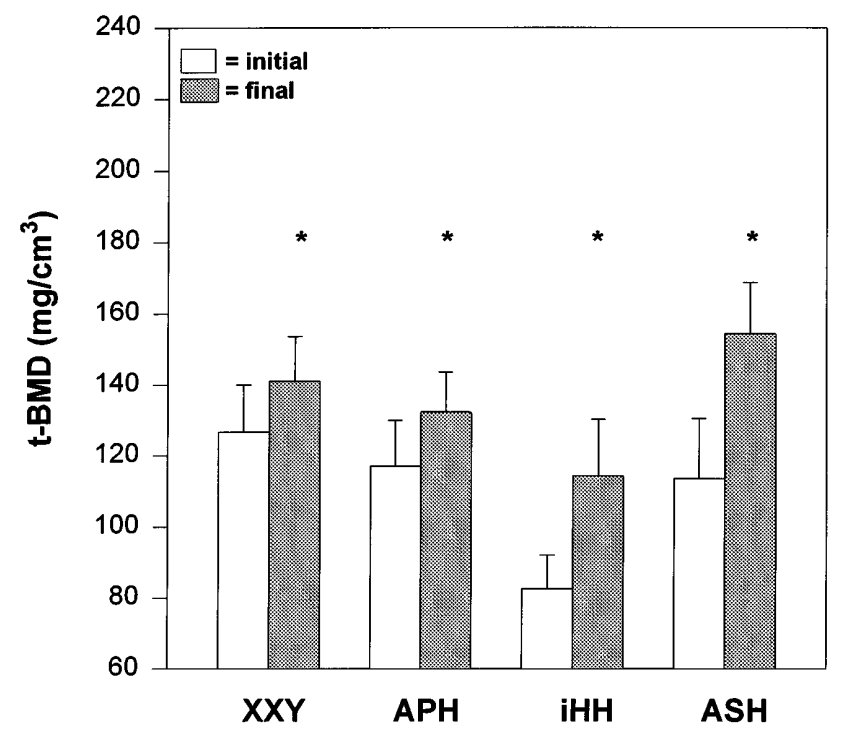

Figure 2 tBMD in 32 men with four classified types of hypogonadism. Values are given as mean \pm S.E.M. per group. Open bars represent the initial values and filled bars represent the final values after $3.2 \pm 1.7$ years of testosterone treatment. ${ }^{*} P<0.05$ compared with initial value (Student's paired $t$-test).

the vertebral midplane level (MVA) was correlated with weight $(0.68 ; P<0.001)$. No correlation was found between the PMA and the MVA, initial serum testosterone levels or age. This could be confirmed if partial correlation was controlled for weight and height. If the influence of weight and age was eliminated by partial correlation, a moderate correlation could be found between muscle area and tBMD $(r=0.44 ; P<0.05)$ but only a tendency towards correlation was seen between muscle area and cBMD $(r=0.27 ; P=0.1)$.

\section{Changes with therapy}

Serum testosterone rose to normal levels in all patients after replacement therapy $(5.4 \pm 1.35$ to $30.1 \pm$ $3.35 \mathrm{nmol} / \mathrm{l})$.

The mean increase of $30 \pm 7 \%$ for tBMD $(P<0.001)$ and $9 \pm 3 \% \quad(P<0.001)$ for $\mathrm{cBMD}$ of the spine over $3.2 \pm 1.7$ years could be confirmed by statistical testing.

Figure $1 \mathrm{a}$ and $\mathrm{b}$ shows the variation with age in initial and final values for $\mathrm{tBMD}$ and $\mathrm{CBMD}$ respectively $(r=-0.39$ and $P<0.05$ for tBMD; $r=-0.35$ and $P<0.05$ for $c B M D)$. For both, there is a significant shift towards age-matched control values (18) during the period of treatment $(P<0.0001)$. The increase remained significant if controlled for body weight.

The individual increase per group of patients is shown in Figs 2 and 3. Each group shows a significant increase in BMD $(P<0.05)$ independent of the type of hypogonadism.

Figure 4 shows the individual increase in PMA for each patient according to the type of hypogonadism.

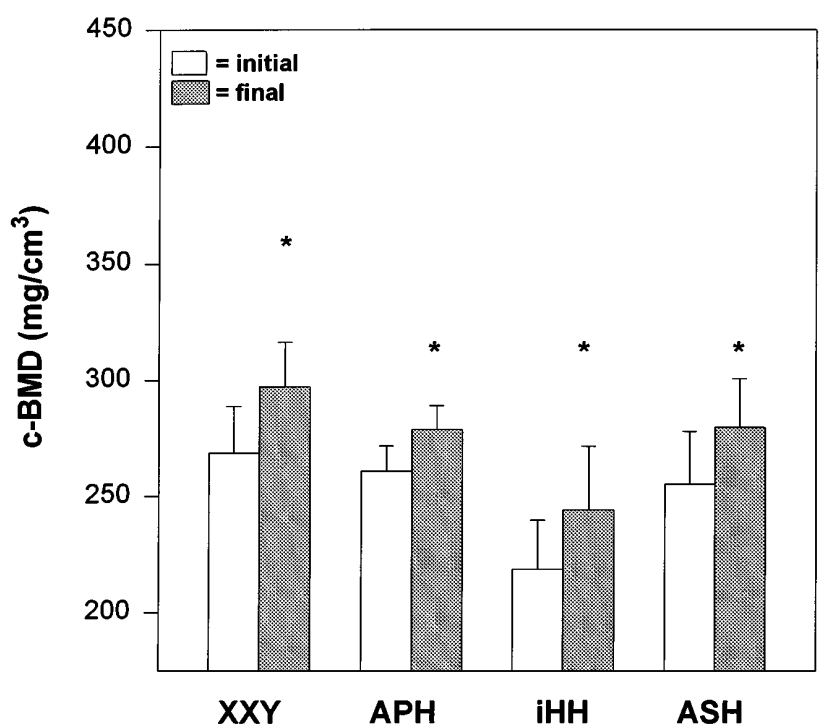

Figure $3 \mathrm{cBMD}$ in 32 men with four classified types of hypogonadism. Values are given as mean \pm S.E.M. per group. Open bars represent the initial values and filled bars represent the final values after $3.2 \pm 1.7$ years of testosterone treatment. ${ }^{*} P<0.05$ compared with initial value (Student's paired $t$-test).

The increase in muscle mass is highest in those with the lowest baseline value and reaches significance $(P<0.05)$ only in patients with $\mathrm{IHH}$ and ASH. Combining all groups, a mean increase of $10 \pm 2.4 \%$ in PMA was found $(P<0.01)$ and remained significant if controlled for body weight. The change in PMA did correspond

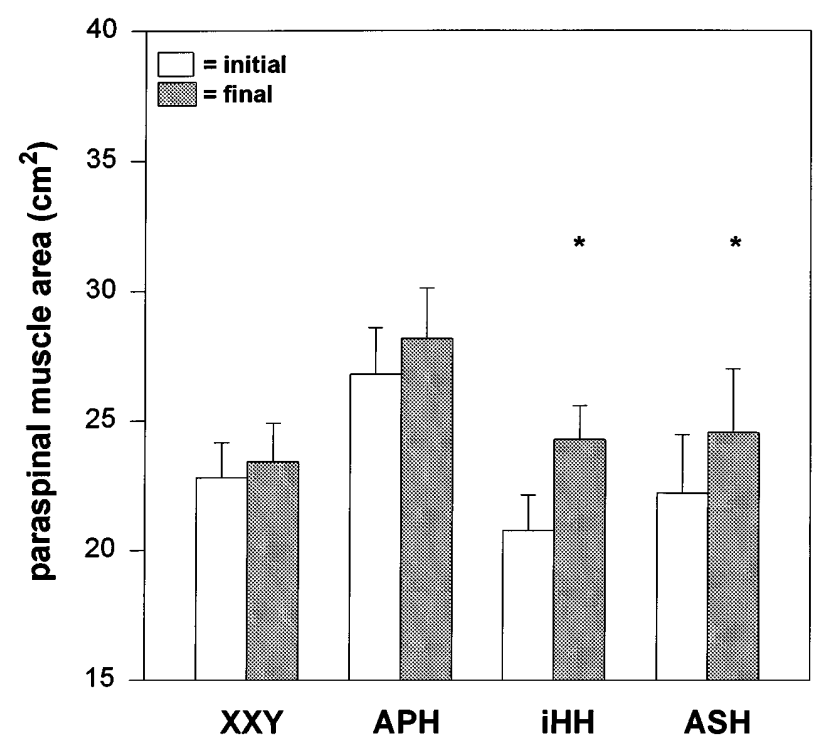

Figure 4 PMA in 32 men with four classified types of hypogonadism. Values are given as mean \pm S.E.M. per group. Open bars represent the initial values and filled bars represent the final values after $3.2 \pm 1.7$ years of testosterone treatment. ${ }^{*} P<0.05$ compared with initial value (Student's paired $t$-test). 


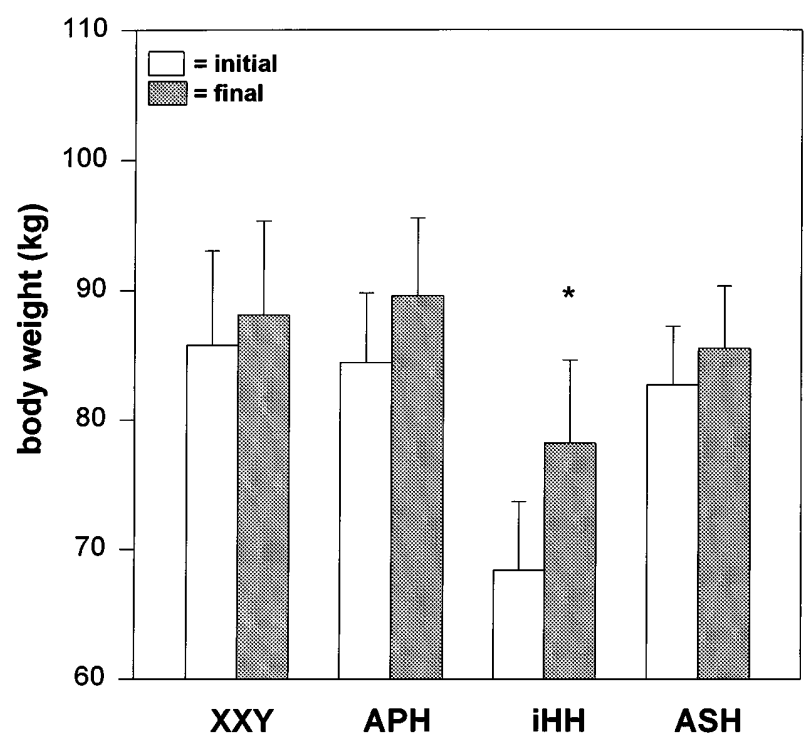

Figure 5 Body weight in 32 men with four classified types of hypogonadism. Values are given as mean \pm S.E.M. per group. Open bars represent the initial values and filled bars represent the final values after $3.2 \pm 1.7$ years of testosterone treatment. ${ }^{*} P<0.05$ compared with initial value (Student's paired $t$-test).

to the change in body weight (Fig. 5$)(r=0.35 ; P<0.05)$ but did not, however, correlate with changes in BMD even when height, initial serum testosterone or age were controlled by partial correlation.

As shown in Fig. 6, no group, not even individual patients with small vertebral area and early-onset hypogonadism, showed any change in MVA over the time of therapy (linear regression between initial and final values: $y=0.97 x+0.38 ; P<0.0001$, not shown).

\section{Discussion}

The results show that effective replacement therapy in hypogonadal men leads to an increase in both tBMD and CBMD of the spine. Osteopenia has been reported in men with Klinefelter syndrome (26), men with idiopathic hypogonadotropic hypogonadism (27), hypogonadism associated with hyperprolactinaemia (28) and after orchidectomy (29). Previous reports have also shown beneficial effects of replacement therapy with testosterone, human choriogonadotrophin or GnRH on bone of men with different types of hypogonadism $(3,4)$. The lower initial BMD and vertebral area found in men with IHH compared with other forms of hypogonadism indicate that the onset and duration of androgen deficiency may be crucial determinants of the extent of reduction in bone mass. In addition to loss of bone mass in hypogonadal men, bone accretion is also impaired in men with pubertal onset of hypogonadism (30).

A correlation between serum testosterone levels and BMD has been reported by some investigators $(26,31)$ but not by others $(4,27)$. These discrepancies may be due to the fact that those studies that did not demonstrate a correlation included patients who had already been treated (4). Indeed, the serum testosterone levels, although indicative of the diagnosis, do not reflect duration and severity of the hypogonadism. In men with acquired forms of hypogonadism it can be difficult to evaluate the onset of androgen deficiency retrospectively. This is supported by our results showing that $\mathrm{BMD}$ is correlated only in men with a congenital type of hypogonadism, either IHH or XXY, before evaluation. Thus serum testosterone levels alone will yield little information about bone status in hypogonadal patients unless the clinical background is taken into consideration. BMD is a more integrative indicator of the duration

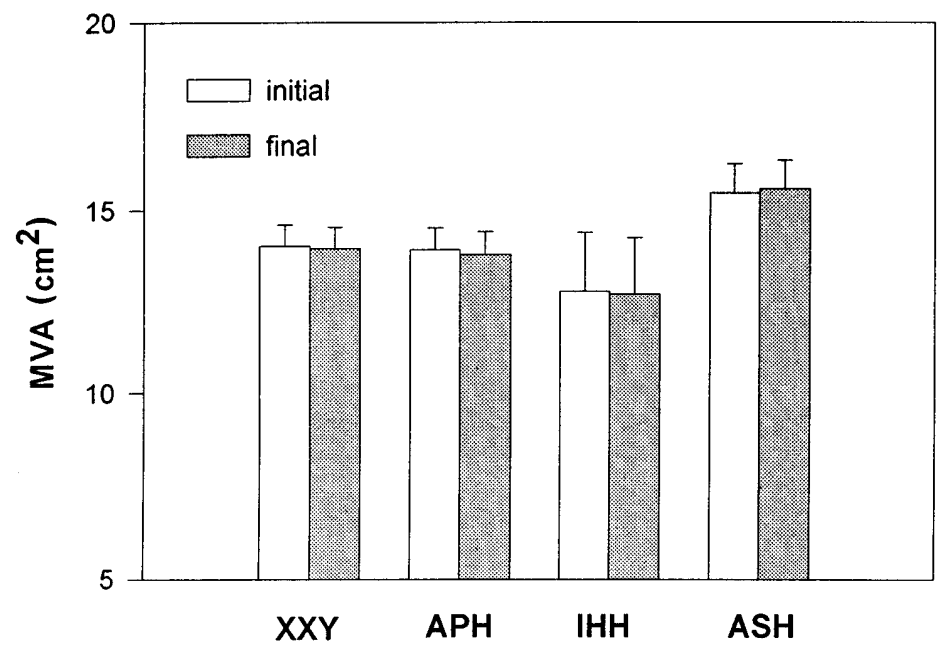

Figure 6 Midvertebral body area (MVA) of 32 hypogonadal men. Values are given as mean \pm S.E.M. per group. Open bars represent initial values and closed bars represent final values after $3.2 \pm 1.7$ years of testosterone treatment. 
and severity of hypogonadism than sporadic serum testosterone levels.

The increase in tBMD and $\mathrm{CBMD}$ observed in patients older than 40 years indicates that age should not be regarded as a limiting factor for the effectiveness of replacement therapy on bone. This confirms a similiar suggestion made in a recent report (32). Even cortical bone, which may react more slowly than trabecular bone, increased under therapy independently of age. A previous study found no increase in tBMD of the spine in men aged 26-52 years with $\mathrm{IHH}$, whereas a slight increase in appendicular bone was seen (3). This might be due to the fact that these patients had received testosterone replacement therapy before the start of the study, whereas our patients had not. Furthermore, appendicular bone, which probably increases in the course of effective therapy, might also respond to a greater extent to physical activity than does spinal bone. Our results suggest that trabecular bone exhibits a greater treatment response than cortical bone of the spine, supporting the general concept that trabecular bone is metabolically more active than cortical bone (33). The fat error has been discussed as a limiting factor in the accuracy of tBMD determination by SE-QCT (32). A recent study has shown that the percentage of body fat decreased $14 \%$ in hypogonadal men during testosterone replacement therapy (8). Glüer et al. (34) showed that a change in $10 \%$ fat per volume vertebral body will lead to a change in $7 \mathrm{mg}$ density equivalents by SE-QCT. Such a change cannot explain the magnitude of increase we found during therapy. In addition, it is hard to believe that BMD increases only within the cortical compartment in which the fat error can be neglected and not within the trabecular compartment of the spine. However, the 'fat error' should be taken into account when considering the 'true' change in trabecular BMD shown by SE-QCT measurements (35). Provided that fat marrow changes to the same extent as subcutaneous fat under testosterone therapy, the mean percentage change observed for tBMD in this study has to be reduced from 30 to about 25 .

Cross-sectional analysis of data confirmed that cBMD and $\mathrm{tBMD}$ declines with age in healthy men, as had been shown by previous reports (19). The correlation found between $\mathrm{tBMD}$ and $\mathrm{CBMD}$ agrees with a study performed on healthy women (36) and is demonstrated here for the first time in hypogonadal men undergoing replacement therapy. Loss of BMD is not, however, the only factor contributing to fracture risk. Studies have reported a wide overlap between BMD values of patients with fractures and those without (37). Bone dimension also determines compressive strength (12) and might have implications for fracture risk. It has been reported that for example women with vertebral fractures show smaller vertebral bone area than those without fractures (13). In addition, differences in bone size, not BMD, have been widely discussed as being responsible for the higher incidence of fractures found in elderly women compared with elderly men (10). An increase in the vertebral area during pubertal growth and in men with age has been suggested on the basis of crosssectional data $(11,22)$. Therefore it is important to assess bone size in addition to BMD as parameters of biomechanical strength and estimate for fracture risk. This may become especially true in patients with a history of pubertal onset of hypogonadism.

Serial investigations on the cross-sectional area of vertebrae of hypogonadal men had not been performed before this study. We did not find any change in the vertebral area of hypogonadal men after initiation of replacement therapy, at least over a period of 3 years. The minimal variation between the follow-up values reflects the precision of the lumbar QCT with respect to the localisation of serial measurements. IHH is associated with pubertal onset, whereas the onset of hypogonadism in the other groups may be variable and could not be ascertained by anamnesis. As seen in men with IHH, early-onset hypogonadism may be associated with small vertebral areas and, because no change is seen with replacement therapy, may imply high risk for vertebral fractures in later life. This highlights the importance of early treatment in boys when puberty fails to occur.

The correlation found between anthropometric parameters (such as height and weight) with the crosssectional area of the vertebrae and the paraspinal muscle is in line with findings from healthy men $(15,37)$, which confirms the accuracy of our measurements. The correlation found between PMA and tBMD, although only weak, may indicate the importance of biomechanical determinants for $\operatorname{BMD}(15,16)$.

Our study shows that PMA increases slightly with testosterone replacement therapy. This finding confirms previous studies showing that replacement therapy in hypogonadal men leads to an increase in lean body and muscle mass $(6-8)$. The relatively large increase seen in the study by Brodsky et al. (6) might be due to the fact that appendicular muscle was assessed. These muscles are more activity-dependent than the paraspinal muscles, which are weight-bearing muscles and may be under more constant strain. This is supported by the correlation we found between PMA and body weight and the relative changes in PMA and body weight respectively.

The increase seen in BMD might reflect a direct effect of testosterone or its metabolites on bone metabolism. In vitro studies reporting the occurrence of androgen receptors (38), androgen metabolism (39) and androgen effects (40) on human osteoblastic cells support this conclusion. A recent study has demonstrated a change in biochemical serum markers of bone metabolism in response to testosterone replacement therapy, suggesting a reduction in bone remodelling (8). In order to monitor treatment response, densitometry might provide information after 1 or 2 years of treatment. Biochemical markers could be earlier indices of treatment 
response if their short-term change is correlated with the long-term effects seen for BMD. Studies combining bone mass measurements with assessment of biochemical markers of bone turnover are therefore necessary. However, BMD and not biochemical markers of bone metabolism is linked to fracture risk as it is emphasized by the generally accepted definition of osteoporosis (41).

A number of conclusions are thus possible from our study. (a) Both trabecular and cortical bone of the spine are affected by androgen deficiency and benefits from effective replacement therapy. (b) Type and age of hypogonadism does not limit the effectiveness of therapy. (c) Men with early-onset hypogonadism may be at greater risk of fracture because of reduced crosssectional area of the vertebrae. (d) Paraspinal muscles might also benefit from effective therapy.

\section{References}

1 Seeman E, Melton LJ, O`Fallon WM \& Riggs BL. Risk factors for spinal osteoporosis in men. American Journal of Medicine 198375 77-83.

2 Ballie SP, Davison CE, Johnson FJ \& Francis RM. Pathogenesis of vertebral crush fractures in men. Age Ageing 199221 139-141.

3 Finkelstein JS, Klibanski A, Neer RM, Doppelt SH, Rosenthal DI, Segre GV \& Crowley WF. Increases in bone density during treatment of men with idiopathic hypogonadotropic hypogonadism. Journal of Clinical Endocrinology and Metabolism 198969 776.

4 Devogelaer JP, De Cooman De S \& de Deuxchaisnes CN Low bone mass in hypogonadal males. Effects of testosterone substitution therapy, a densitometric study. Maturitas 199215 17-23.

5 Ariska O, Ariska M, Hosaka A, Shimura N \& Yabuta K. Effect of testosterone on radial BMD in adolescent male hypogonadism. Acta Paediatrica Scandinavia $199180378-380$.

6 Brodsky IG, Balagopal P \& Nair SK. Effects of testosterone replacement on muscle mass and protein synthesis in hypogonadal men: a clinical research center study. Journal of Clinical Endocrinology and Metabolism 199681 3469-3475.

7 Wang C, Eyre DR, Clark R, Kleinberg C, Newman C, Iranmanesh A, Velduis J, Dudley RE, Berman N, Davidson T, Barstow TJ, Sinow R, Alexander G \& Swerdloff RS. Sublingual testosterone replacement improves muscle mass and strength, decreases bone resorption, and increases bone formation markers in hypogonadal men: a clinical research center study. Journal of Clinical Endocrinology and Metabolism 199681 3654-3662.

8 Katznelson L, Finkelstein JS, Schoenfeld DA, Rosenthal DI, Anderson E \& Klibanski A. Increase in bone density and lean body mass during testosterone administration in men with acquired hypogonadism. Journal of Clinical Endocrinology and Metabolism $1996814358-4365$.

9 Finkelstein JS \& Klibanski A. Effects of androgens on bone metabolism. In Testosterone: Action, Deficiency, Substitution, pp 115-135. Eds E Nieschlag \& HM Behre. Berlin: Springer-Verlag, 1990.

10 Orwoll ES \& Klein RF. Osteoporosis in men. Endocrine Reviews $19951687-116$.

11 Moseklide L \& Moseklide L. Normal vertebral body size and compressive strength: relations to age and to vertebral and iliac trabecular bone compressive strength. Bone $19867207-212$

12 Brinckmann P, Biggemann M \& Hilweg D. Prediction of the compressive strength of human lumbar vertebrae. Clinical Biomechanics 19894 (Suppl 2) 1.

13 Gilsanz V, Shultz EE \& Loro L. Smaller cross-sectional area of intact vertebral bodies in women with vertebral fractures. Bone Mineral Research 19938 326-331.
14 Colletti LA, Edwards J, Bordon L, Shary J \& Bell NH. The effects of muscle-building exercise on BMD of the radius, spine, and hip in young men. Calcified Tissue International 198945 12-14.

15 Block JE, Friedlander AL, Brooks GA, Steiger P, Stubbs H \& Genant HK. Determinants of bone density among athletes engaged in weight-bearing and non-weight-bearing activity. Journal of Applied Physiology 198967 1100-1105.

16 Nordström P, Thorsen K, Bergström E \& Lorentzon R. High bone mass and altered relationships between bone mass, muscle strength, and body constitution in adolescent boys on a high level of physical activity. Bone 199619 189-195.

17 Nieschlag E \& Behre HM. Pharmacology and clinical use of testosterone. In Testosterone: Action, Deficiency, Substitution, pp 92-115. Eds E Nieschlag \& HM Behre. Berlin: SpringerVerlag, 1990.

18 Kliesch S, Behre HM \& Nieschlag E. High efficacy of gonadotropin or pulsatile GnRH treatment in hypogonadotropic hypogonadal men. European Journal of Endocrinology $1994131347-354$.

19 Kalender AW, Felsenberg D, Louis O, Lopez P, Klotz E, Osteaux M \& Fraga J. Reference values for trabecular and cortical vertebral bone density in single and dual-energy quantitative computed tomography. European Journal of Radiology 1989 9 75-80.

20 Cann CE \& Genant HK. Precise measurement of vertebral mineral content using computed tomography. Journal of Computer Assisted Tomography 19804 493-500.

21 Louis O, Luypaert R, Kalender WA \& Osteaux M. Reproducibility of CT bone densitometry: operator versus automated ROI definition. European Journal of Radiology 19888 82-84.

22 Gilsanz V, Boechat MI, Gilsanz R, Loro ML, Roe TF \& Goodman WG. Gender differences in vertebral body sizes in children and adolescents. Radiology $1994190673-677$.

23 Gilsanz V, Boechat MI, Roe TF, Loro ML, Sayre JW \& Goodman WG. Gender differences in vertebral sizes in adults: biomechanical implications. Radiology $1994190678-682$.

24 Steiger P, Block JE, Friedlander A \& Genant HK. Precise determination of paraspinous musculature by quantitative computed tomography. Journal of Computer Assisted Tomography 198812 616-620.

25 Chandolia RK, Weinbauer GF, Simoni M, Behre HM \& Nieschlag E. Comparative effects of chronic administration of the non-steroidal antiandrogens flutamide and casodex on the reproductive system of the adult male rat. Acta Endocrinologica 199115 17-23.

26 Foresta C, Ruzza G, Mioni R, Meneghello A \& Baccichetti B. Testosterone and bone loss in Klinefelter Syndrome. Hormone and Metabolic Research 198315 56-57.

27 Finkelstein JS, Klibanski A, Neer RM, Greenspan SL, Rosenthal DI \& Crowley Jr WF. Osteoporosis in men with idiopathic hypogonadotropic hypogonadism. Annals of Internal Medicine 1987106 354-361.

28 Greenspan SL, Oppenheim DS \& Klibanski A. Importance of gonadal steroids to bone mass in men with hyperprolactinemic hypogonadism. Annals of Internal Medicine 1989110 526-531.

29 Stephan JJ, Lachman M, Zvevina J, Pacovsky V \& Baylink D. Castrated men exhibited bone loss: effect of calcitonin treatment on biochemical indices of bone remodelling. Journal of Clinical Endocrinology and Metabolism 198969 523-527.

30 Finkelstein JS, Klibanski A \& Neer RM. A longitudinal evaluation of BMD in adult men with histories of delayed puberty. Journal of Clinical Endocrinology and Metabolism 199681 1152-1155.

31 Isaia G, Mussetta M, Pecchio F, Sciolla A, di Stefano M \& Molinatti GM. Effect of testosterone on bone in hypogonadal males. Maturitas $19921547-51$.

32 Aspray TJ, Francis RM, Rutter M \& Walker M. Consequences of withholding testosterone. Letter. Lancet 199614609.

33 Vanderschueren D \& Bouillon R. Androgens and bone. Calcified Tissue International $199556341-346$.

34 Glüer CC, Reiser UJ, Davis CA, Rutt BK \& Genant HK. Vertebral mineral determination by quantitative computed tomography (QCT): accuracy of single and dual energy computed measurements. Journal of Computer Assisted Tomography $198812242-258$. 
35 Pacifici R, Rupich S \& Avioli LV. Vertebral cortical bone mass measurement by a new quantitative computer tomography method. Correlations with vertebral trabecular bone measurements. Calcified Tissue International 199047 215-220.

36 Kaplan FS, Dalinka M, Karp JS, Fallon MD, Katz M, Boden S, Simpson E, Attie M \& Hadded JG. Quantitative computed tomography reflects vertebral fracture morbidity in osteopenic patients. Computed Tomography 198912 949-955.

37 Ito M, Hayashi K, Uetani M, Yamada M, Ohki M \& Nakamura T. Association between anthropometric measures and spinal bone density. Investigative Radiology 199429 812-817.

38 Colvard DS, Eriksen EF, Keeting PE, Wilson EM, Lubahn DB, French FS et al. Identification of androgen receptors in norma human osteoblast-like cells. Proceedings of the National Academy of Sciences of the USA $198986854-857$.
39 Schweikert H-U, Wolf L \& Romalo G. Oestrogen formation from androstenedione in human bone. Clinical Endocrinology 199543 37-42.

40 Kasperk CH, Wegedal JE, Farley JR, Linkhart TA, Turner RT \& Baylink DJ. Androgens directly stimulate proliferation of bone cells in vitro. Endocrinology 1989124 1576-1578.

41 Kanis JA, Melton LJ III, Christianson C, Johnston CC \& Khaltev N. The diagnosis of osteoporosis. Journal of Bone and Mineral Research $199491137-1141$.

Received 27 January 1997

Accepted 26 August 1997 